\title{
Serum Lactate Dehydrogenase Before Nivolumab Treatment Could Be a Therapeutic Prognostic Biomarker for Patients With Metastatic Clear Cell Renal Cell Carcinoma
}

\author{
SUGURU SHIROTAKE ${ }^{1}$, KIMIHARU TAKAMATSU ${ }^{2}$, RYUICHI MIZUNO ${ }^{2}$, GO KANEKO ${ }^{1}$, \\ KOSHIRO NISHIMOTO $^{1}$, MOTOTSUGU OYA ${ }^{2}$ and MASAFUMI OYAMA ${ }^{1}$ \\ ${ }^{1}$ Department of Uro-Oncology, Saitama Medical University International Medical Center, Hidaka, Japan; \\ ${ }^{2}$ Department of Urology, Keio University School of Medicine, Tokyo, Japan
}

\begin{abstract}
Background/Aim: The purpose of this retrospective study was to identify the predictive biomarkers of response to pretreatment for patients with metastatic renal cell carcinoma (mRCC) treated with nivolumab. Materials and Methods: The subjects were 54 patients treated with nivolumab for $m R C C$ with a clear cell component (mccRCC) between September 2016 and February 2018. We analyzed the impact of serum biomarkers (lactate dehydrogenase [LDH], neutrophil-to-lymphocyte ratio, and C-reactive protein) on patients treated with nivolumab. We adopted the International Metastatic Renal Cell Carcinoma Database Consortium prognostic model using six clinical factors ( $0=$ favorable, 1 or 2=intermediate, 3 to $6=$ poor risk groups, respectively). Results: The prognostic risk classification (nonpoor vs. poor) and serum LDH levels were correlated with the objective response of nivolumab treatment for mccRCC. Elevated serum LDH levels at baseline were an independent biomarker for progression-free survival (PFS) of mccRCC patients receiving nivolumab [HR=2.268 (95\%CI=1.014-5.051), $p=0.046]$. Notably, high LDH levels were associated with a poorer PFS for patients in the favorable-risk group. Conclusion: Serum LDH levels at baseline before nivolumab treatment were associated with the objective response and clinical outcome of nivolumab treatment.
\end{abstract}

Nivolumab is an immune checkpoint inhibitor (ICI). It is a highly selective anti-programmed death 1 (PD-1) human monoclonal IgG4 antibody that potentiates T-cell responses

\footnotetext{
Correspondence to: Suguru Shirotake, Department of UroOncology, Saitama Medical University International Medical Center, 1397-1 Yamane, Hidaka, Saitama, Japan. Tel: +81 429844111, Fax: +81 429844569, e-mail: ss6001@5931.saitamamed.ac.jp
}

Key Words: Renal cell carcinoma, nivolumab, outcome, lactate dehydrogenase. by blocking the binding of PD-1 on activated T cells with its ligands, PD-L1 and PD-L2, expressed on antigenpresenting cells and on some tumor cells (1). Advanced and metastatic renal cell carcinomas (mRCC) are currently treated with molecular targeted therapy (MTT) agents as a first-line standard of care (2). In a phase III trial [CheckMate 025 trial (3)], the overall survival (OS) of patients treated with nivolumab was significantly prolonged compared to those treated with everolimus for metastatic clear cell RCC (mccRCC) patients who had failed prior MTT. Furthermore, various trials have reported excellent results using combination therapy of MTTs and /or ICIs for $\operatorname{mccRCC}(4,5)$.

Despite these clinical successes, ICI for mccRCC does not achieve long-lasting responses for all patients. BuderBakhaya et al. have reviewed the current status of prognostic and predictive biomarkers (including clinical, tissue, blood, and stool biomarkers, as well as imaging biomarkers) in ICI mainly for melanoma and other malignancies (6). Blood biomarkers such as serum lactate dehydrogenase (LDH), C-reactive protein (CRP), and the neutrophil-to-lymphocyte ratio (NLR), have been identified as possible biomarkers for response to ICI (6). However, there is only a small number of reports on mRCC patients. The purpose of this retrospective study was to identify the predictive biomarkers at pretreatment in terms of response of patients with mccRCC treated with nivolumab.

\section{Materials and Methods}

Ethics and patient selection. This retrospective study received approval from the Institutional Review Board of Saitama Medical University International Medical Center (SIMC, approval \#: 14049). SIMC $(n=31)$ and Keio University School of Medicine $(n=23)$ provided data on 54 patients treated with nivolumab for mRCC with a clear cell component (mccRCC) between September 2016 and February 2018. Table I shows a comparison of patient characteristics. 
Routine examination and follow-up. In order to predict the outcomes of mccRCC patients at the initiation of first line therapy, we stratified them into three groups using six factors (time from RCC diagnosis to systemic therapy initiation, Karnofsky performance status (KPS), anemia, neutrophilia, thrombophilia, and serum corrected calcium) based on the International Metastatic Renal Cell Carcinoma Database Consortium (IMDC) model ( $0=$ favorable, 1 or $2=$ intermediate, 3 to $6=$ poor risk groups, respectively) (7). Basically, patients with $\mathrm{mRCC}$ who had failed MTTs received intravenously $3 \mathrm{mg} / \mathrm{kg}$ nivolumab, every two weeks. Laboratory values were standardized according to the institutional upper and lower limits of normal values. Radiographic evaluations using computed tomography (CT) and/or magnetic resonance imaging (MRI) were performed every two to three months, while additional CT and elective bone scans were performed when clinically indicated. Patients without neurological symptoms were generally not subjected to brain imaging tests.

Clinical outcome. Overall survival (OS) was defined as the time from initiation of first line targeted therapy to the date of death from any cause or date of censorship from last follow up. Progressionfree survival (PFS) was defined as the time between nivolumab treatment initiation and radiographical progression or cancer-related death. Based on the Response Evaluation Criteria in Solid Tumors (RECIST) version 1.1 (8), we originally classified patients into two groups: A responder group of patients with complete response (CR), partial response (PR), or long stable disease (SD) for over 6 months and a non-responder group with progression disease (PD) or short SD for less than 6 months.

Statistical analysis. The variables of different groups were compared using the chi-square test or the Mann-Whitney $U$-test as appropriate. Laboratory values were standardized according to the institutional upper and lower limits of normal values. Values were presented as medians and interquartile ranges (IQR) or confidence intervals $(\mathrm{CI})$ for continuous variables and as frequencies with percentages for categorical variables. Survival curves were constructed using the Kaplan-Meier method and compared using the log-rank test. Univariate and multivariate analyses were performed using the Cox proportional hazards model to identify factors associated with PFS. The level of significance was set at $p<0.05$. The above analyses were done using the SPSS version 23.0 statistical software package.

\section{Results}

Patient characteristics (Table I). Fifty-four cases (44 males, 10 females) were treated with nivolumab for mccRCC in our cohort. The median follow-up duration from nivolumab initiation was 10.6 months (IQR=7.5-14.3). At initial systemic treatment for mccRCC, the status of IMDC risk classification was favorable in $12(22.2 \%)$, intermediate in $34(63.0 \%)$, and poor in eight patients (14.8\%), respectively. Prior nephrectomy was performed in 49 patients $(90.7 \%)$, and prior MTTs with more than three agents before nivolumab treatment was performed in 13 patients $(24.1 \%)$.

During the first nivolumab treatment, the median age was
69 years (IQR=63-75), and 10 patients $(18.5 \%)$ had KPS less than $80 \%$. The metastatic sites were the lungs in $40(74.1 \%)$, liver in $13(24.1 \%)$, bone in $15(27.8 \%)$, lymph nodes in 14 $(25.9 \%)$, brain in six $(11.1 \%)$, and pancreas in seven patients $(13.0 \%)$, respectively. The median values of laboratory data were 2.89 (IQR=1.85-4.61) for NLR, $196 \mathrm{U} / \mathrm{l}$ (IQR=164245) for $\mathrm{LDH}$, and $0.67 \mathrm{mg} / \mathrm{dl}(\mathrm{IQR}=0.19-1.91)$ for CRP, respectively.

Clinical outcome (Table I, Figure 1A-C). Best objective responses following nivolumab treatment were $\mathrm{CR}$ in two (3.7\%), PR in 17 (31.5\%), SD in $23(42.6 \%)$, and PD in 12 patients $(22.2 \%)$, respectively (Table I). The median PFS in the patients with SD was 6.0 months. During follow-up, 10 patients died, and 28 patients had disease progression. The median OS objective in the total cohort $(n=54)$ was not reached (Figure 1A), but the median PFS was 7.9 months (95\% CI=3.6-12.3, Figure 1B). In the sub-group analysis, the median PFS according to IMDC risk classification was 6.2 $(95 \% \mathrm{CI}=1.6-10.8)$ in the favorable risk group, 11.3 (5.4$17.3)$ in the intermediate risk group, and 1.8 months (1.0-2.7) in the poor risk group, respectively (Figure 1C).

Nivolumab treatment responders versus non-responders (Table I). We distinguished patients with SD into long and short SD groups based on the median PFS (6.0 months), and then we classified all patients into two groups [A responder group $(n=31)$ : $C R, P R$, and a long $S D$ and a non-responder group $(n=23)$ : PD and short SD groups] as mentioned previously (see Material and Methods). Regarding IMDC risk classification, the responders included fewer patients with favorable risk along with poor risk patients and many of the intermediate risk group patients compared with the non-responders (favorable: 16.1 vs. 30.4\%, intermediate: $77.4 v s .43 .5 \%$, poor: 6.5 vs. $26.1 \%, p=0.029)$. As for laboratory data at nivolumab initiation, the median LDH levels were significantly lower in the responders than in the non-responders (178vs. $229 \mathrm{U} / 1, p<0.001)$, and similarly NLR in the responders showed a lower tendency (2.46 vs. $3.33, p=0.060$ ). Meanwhile, there were no significant differences in the CRP levels $(p=0.944)$ or other factors (i.e. gender, prior nephrectomy, number of prior MTTs, age, KPS and metastatic sites at nivolumab initiation) between the two groups.

Predictive factors of therapeutic effect with nivolumab treatment (Table II, Figure ID-F). We investigated the predictive factors of PFS in mccRCC patients treated with nivolumab based on the above results that compared the characteristics of the responders and non-responders. Univariate and multivariate analyses were performed using four indices: IMDC risk classification (non-poor $v s$. poor), and NLR, LDH, and CRP (low vs. high, median level as a 
Table I. Patients' characteristics.

\begin{tabular}{|c|c|c|c|c|c|c|c|}
\hline \multirow[t]{2}{*}{ Variables } & \multicolumn{2}{|c|}{ Total cohort $(\mathrm{n}=54)$} & \multicolumn{2}{|c|}{ Responder $(\mathrm{n}=31)$} & \multicolumn{2}{|c|}{ Non-responder $(n=23)$} & \multirow[t]{2}{*}{$p$-Value } \\
\hline & $\mathrm{n}$ & $\%$ & $\mathrm{n}$ & $\%$ & $\mathrm{n}$ & $\%$ & \\
\hline \multicolumn{7}{|l|}{ Gender } & $0.728^{\#}$ \\
\hline Male & 44 & $(81.5)$ & 26 & $(83.9)$ & 18 & $(78.3)$ & \\
\hline Female & 10 & $(18.5)$ & 5 & $(16.1)$ & 5 & $(21.7)$ & \\
\hline \multicolumn{7}{|l|}{ IMDC risk classification } & $0.029^{\# \#}$ \\
\hline Favorable & 12 & $(22.2)$ & 5 & $(16.1)$ & 7 & $(30.4)$ & \\
\hline Intermediate & 34 & $(63.0)$ & 24 & (77.4) & 10 & $(43.5)$ & \\
\hline Poor & 8 & $(14.8)$ & 2 & $(6.5)$ & 6 & $(26.1)$ & \\
\hline \multicolumn{7}{|l|}{ Prior nephrectomy } & $1.000^{\#}$ \\
\hline Yes & 49 & $(90.7)$ & 28 & $(90.3)$ & 21 & $(91.3)$ & \\
\hline No & 5 & $(9.3)$ & 3 & $(9.7)$ & 2 & $(8.7)$ & \\
\hline \multicolumn{7}{|l|}{ Prior MTTs } & $0.620^{\# \#}$ \\
\hline 1 & 23 & $(42.6)$ & 12 & $(38.7)$ & 11 & $(47.8)$ & \\
\hline 2 & 18 & $(33.3)$ & 12 & $(38.7)$ & 6 & $(26.1)$ & \\
\hline$\geq 3$ & 13 & $(24.1)$ & 7 & $(22.6)$ & 6 & $(26.1)$ & \\
\hline \multicolumn{8}{|c|}{ Status at the first nivolumab treatment } \\
\hline Age (median, IQR, years old) & $69(63-75)$ & & $71(65-76)$ & & $67(57-74)$ & & $0.106^{\# \# \#}$ \\
\hline KPS $(\%)$ & & & & & & & $0.407^{\# \#}$ \\
\hline 100 & 33 & $(61.1)$ & 21 & $(67.7)$ & 12 & $(52.2)$ & \\
\hline 90 & 11 & $(20.4)$ & 6 & $(19.4)$ & 5 & $(21.7)$ & \\
\hline$\leq 80$ & 10 & $(18.5)$ & 4 & $(12.9)$ & 6 & $(26.1)$ & \\
\hline \multicolumn{8}{|l|}{ Metastatic sites } \\
\hline Lung & 40 & $(74.1)$ & 24 & $(77.4)$ & 16 & $(69.6)$ & $0.515^{\# \#}$ \\
\hline Liver & 13 & $(24.1)$ & 6 & $(19.4)$ & 7 & $(30.4)$ & $0.346^{\# \#}$ \\
\hline Bone & 15 & $(27.8)$ & 8 & $(25.8)$ & 7 & $(30.4)$ & $0.707^{\# \#}$ \\
\hline Lymph node & 14 & $(25.9)$ & 9 & $(29.0)$ & 5 & $(21.7)$ & $0.545^{\# \#}$ \\
\hline Brain & 6 & $(11.1)$ & 2 & $(6.5)$ & 4 & $(17.4)$ & $0.384^{\#}$ \\
\hline Pancreas & 7 & $(13.0)$ & 5 & $(16.1)$ & 2 & $(8.7)$ & $0.685^{\#}$ \\
\hline \multicolumn{8}{|l|}{ Laboratory data (median, IQR) } \\
\hline Neutrophil (\%) & $66.6(58.3-73.9)$ & & $63.4(54.8-72.3)$ & & $69.1(62.9-80.0)$ & & $0.074^{\# \# \#}$ \\
\hline Lymphocyte (\%) & $23.6(15.8-31.1)$ & & $26.4(20.5-31.9)$ & & $20.6(14.2-27.7)$ & & $0.061^{\# \# \#}$ \\
\hline NLR & $2.89(1.85-4.61)$ & & $2.46(1.74-3.61)$ & & $3.33(2.27-5.68)$ & & $0.060^{\# \# \#}$ \\
\hline LDH (U/l) & $196(164-245)$ & & $178(147-204)$ & & $229(195-334)$ & & $<0.001^{\# \# \#}$ \\
\hline $\mathrm{CRP}(\mathrm{mg} / \mathrm{dl})$ & $0.67(0.19-1.91)$ & & $0.79(0.25-1.37)$ & & $0.51(0.07-3.57)$ & & $0.944^{\# \# \#}$ \\
\hline \multicolumn{8}{|l|}{ Best objective response } \\
\hline $\mathrm{CR}$ & 2 & $(3.7)$ & 2 & $(6.5)$ & 0 & $(0.0)$ & \\
\hline PR & 17 & $(31.5)$ & 17 & $(54.8)$ & 0 & $(0.0)$ & \\
\hline SD & 23 & $(42.6)$ & 12 & $(38.7)$ & 11 & $(47.8)$ & \\
\hline PD & 12 & $(22.2)$ & 0 & $(0.0)$ & 12 & $(52.2)$ & \\
\hline
\end{tabular}

IMDC: International metastatic renal cell carcinoma database consoritum; MTTs: molecular targeted therapies; IQR: interquartile range; KPS: Karnofsky performance status; NLR: neutrophil-to-lymphocyte ratio; LDH: lactate dehydrogenase; CRP: C-reactive protein; CR: complete response; PR: partial response; SD: stable disease; PD: progression disease; responder: PR, CR or SD (PFS>6.0 mo); non-responder: PD, SD (PFS $\leq 6.0$ mo). \#Fisher, \#\#Pearson, \#\#\#Mann-Whitney $U$-test.

reference). As a result, serum LDH levels before nivolumab treatment was an independent predictive factor of PFS during nivolumab treatment in mccRCC patients $[\mathrm{HR}=2.268(95 \% \mathrm{CI}=1.014-5.051), p=0.046]$. Interestingly, especially in the favorable group, the patients with high LDH [median PFS 3.2 months (95\% CI=1.5-5.0)] had significantly worse PFS with nivolumab treatment than those with low LDH (not reached, $p=0.037$, Figure 1D). There were no significant associations between LDH levels before nivolumab treatment and therapeutic outcome for the intermediate $(p=0.308$, Figure 1E) or poor groups $(p=0.549$, Figure 1F).

\section{Discussion}

The current study showed that IMDC risk classification (nonpoor vs. poor) and serum LDH levels were associated with radiographic objective response to nivolumab treatment for 
Table II. Predictive factors of progression-free survival during nivolumab treatment in mRCC patients.

\begin{tabular}{|c|c|c|c|c|c|c|}
\hline & \multicolumn{3}{|c|}{ Univariate analysis } & \multicolumn{3}{|c|}{ Multivariate analysis } \\
\hline & HR & $95 \% \mathrm{CI}$ & $p$-Value & HR & $95 \% \mathrm{CI}$ & $p$-Value \\
\hline \multicolumn{7}{|c|}{ IMDC risk classification } \\
\hline Non-poor $v s$. poor & 2.740 & $1.101-6.822$ & 0.030 & 1.681 & $0.660-5.366$ & 0.237 \\
\hline \multicolumn{7}{|l|}{ NLR } \\
\hline Low vs. high & 1.957 & $0.912-4.202$ & 0.085 & 1.894 & $0.826-4.348$ & 0.132 \\
\hline \multicolumn{7}{|l|}{$\mathrm{LDH}(\mathrm{U} / \mathrm{l})$} \\
\hline Low $v s$. high & 2.494 & $1.144-5.435$ & 0.021 & 2.268 & $1.014-5.051$ & 0.046 \\
\hline \multicolumn{7}{|l|}{$\mathrm{CRP}(\mathrm{mg} / \mathrm{dl})$} \\
\hline Low vs. high & 1.244 & $0.588-2.628$ & 0.568 & 1.430 & $0.570-3.592$ & 0.446 \\
\hline
\end{tabular}

Median NLR 2.89; median LDH 196.5 U/l; median CRP 0.67 mg/dl.

mccRCC (Table I), and that elevated serum LDH levels at baseline were an independent biomarker for PFS of mccRCC patients receiving nivolumab (Table II); in particular, notably higher LDH levels were associated with worse PFS for patients in the favorable risk group (Figure 1D).

LDH is an enzyme required during the process of turning sugar into energy in human cells. This enzyme is present in many kinds of organs and tissues throughout the body, and is released by rapidly growing tumors. Therefore, serum LDH is often used as an index of organ injury and tumor burden. For melanoma, it has been indicated as a significant prognostic biomarker in the American Joint Committee on Cancer classification (9). Also for mRCC, the Memorial Sloan Kettering Cancer Center (MSKCC) risk model which consists of five prognostic factors including serum $\mathrm{LDH}$ levels at baseline, has generally been adopted for prognostic prediction of mRCC patients in the era of cytokines and MTTs (10). Recently, the IMDC risk model consisting of six prognostic factors excluding serum LDH levels at baseline, has been employed for therapeutic selection, as well as prognostic prediction for mRCC patients in the new era of ICIs $(7,11)$; however, our results suggest that pretreatment LDH levels could be a convenient blood biomarker of response to nivolumab treatment for mccRCC patients.

ICI treatments for mRCC have been approved following major phase III trials: the CheckMate 025 trial (using the MSKCC risk model), nivolumab vs. everolimus as sequential therapy in all risk groups (3); the CheckMate 214 trial (using the IMDC risk model), and nivolumab plus ipilimumab vs. sunitinib as first-line treatment in intermediate and poor risk groups (5). Interestingly, subgroup analyses of OS in both trials consistently showed that the clinical outcomes of ICI treatments in favorable risk groups were far from satisfactory, and our results were similar (median PFS 6.2 in the favorable, 11.3 in intermediate, and 1.8 months in poor group, respectively, Figure 1C). McDermott et al. studied the gene signature related to angiogenesis, immune and antigen presentation, and myeloid inflammation in RCC tumors, and found that highly angiogenic tumors were more responsive to antiangiogenic therapy, but not ICIs (12). High LDH serum levels as an angiogenic biomarker, indicating the overexpression of vascular endothelial growth factor (VEGF) and VEGF receptor (13), could be related to better outcomes in patients undergoing antiangiogenic treatment (14). Recently, the cancer immunogram has been investigated as a framework for personalized immunotherapy, suggesting that LDH levels could be a biomarker of inhibitory tumor metabolism (e.g. intratumoral lactic acid and low $\mathrm{pH}$ ) and impairment of antitumor T-cell function (15). LDH levels at nivolumab initiation could be a therapeutic biomarker for mccRCC patients with favorable risk, who initially have few inflammatory markers.

Regarding other biomarkers, Shrotriya et al. reviewed the role of CRP as a prognosis biomarker for tumor recurrence and treatment response in adult solid tumors including mRCC (16); however, the database was compiled before the introduction of ICIs. Bilen et al. reported an association between NLR at baseline and outcome of mRCC patients receiving nivolumab (17). Among our small cohort, there was no significant association between CRP levels and clinical outcome, but a strong relation was found between NLR and objective response.

The present study has several limitations. First, it was limited by its retrospective nature, as well as by its short median follow-up and limited number of Japanese patients. The laboratory data were collected separately at each institution. Not all patients underwent prior nephrectomy or biopsy before being administered first-line targeted therapy. Furthermore, the systematic therapeutic strategy, such as surgical procedures (radical or cytoreductive), radiotherapy (for bone or brain), and medications (types or timing), were not standardized by the various physicians.

In conclusion, despite the small cohort with heterogeneous characteristics, our results showed that serum LDH levels at 

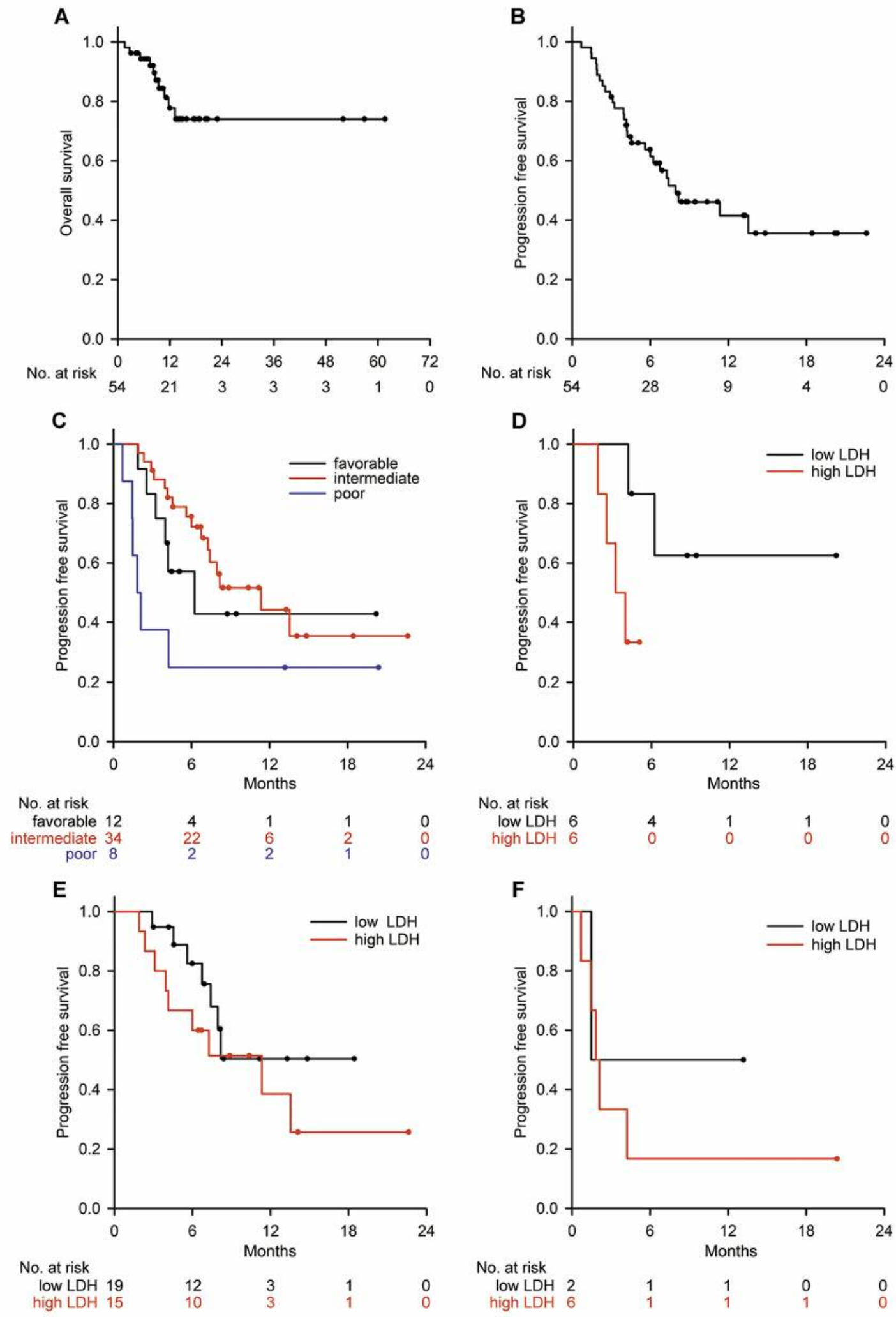

Figure 1. Kaplan-Meier curves showing overall survival (OS, A) and progression-free survival (PFS, B) in the total cohort, as well as PFS according to the IMDC risk classification $(C)$. Kaplan-Meier curves showing the association between PFS and serum LDH levels at baseline of nivolumab treatment for patients in the favorable $(D)$, intermediate $(E)$, and poor $(F)$ risk groups, respectively. 
baseline before nivolumab treatment could be a therapeutic prognostic biomarker for patients with mccRCC. Further study is necessary to identify convenient and effective biomarkers to predict ICIs treatment response in order to select the optimal therapeutic strategy in the new era of ICIs.

\section{Conflicts of Interest}

All Authors declare that they have no conflicts of interest regarding this study.

\section{Authors' Contributions}

Conception and design of the study, Suguru Shirotake and Kimiharu Takamatsu; Collection and assembly of data, Suguru Shirotake, Kimiharu Takamatsu, Ryuichi Mizuno, and Go Kaneko; Analysis and interpretation of data, Suguru Shirotake, Kimiharu Takamatsu and Go Kaneko; Drafting of the article, Suguru Shirotake, Kimiharu Takamatsu and Go Kaneko; Critical revision of the article for important intellectual content, Ryuichi Mizuno, Koshiro Nishimoto, Mototsugu Oya and Masafumi Oyama; Final approval of the article, Suguru Shirotake. All Authors read and approved the version submitted for publication

\section{References}

1 Pardoll DM: The blockade of immune checkpoints in cancer immunotherapy. Nat Rev Cancer 12(4): 252-264, 2012. PMID: 22437870. DOI: $10.1038 / \mathrm{nrc} 3239$

2 Hsieh JJ, Purdue MP, Signoretti S, Swanton C, Albiges L, Schmidinger M, Heng DY, Larkin J and Ficarra V: Renal cell carcinoma. Nat Rev Dis Primers 3: 17009, 2017. PMID: 28276433. DOI: $10.1038 /$ nrdp.2017.9

3 Motzer RJ, Escudier B, McDermott DF, George S, Hammers HJ, Srinivas S, Tykodi SS, Sosman JA, Procopio G, Plimack ER, Castellano D, Choueiri TK, Gurney H, Donskov F, Bono P, Wagstaff J, Gauler TC, Ueda T, Tomita Y, Schutz FA, Kollmannsberger C, Larkin J, Ravaud A, Simon JS, Xu LA, Waxman IM, Sharma P and CheckMate I: Nivolumab versus everolimus in advanced renal-cell carcinoma. N Engl J Med 373(19): 1803-1813, 2015. PMID: 26406148. DOI: $10.1056 /$ NEJMoa 1510665

4 Motzer RJ, Hutson TE, Glen H, Michaelson MD, Molina A, Eisen T, Jassem J, Zolnierek J, Maroto JP, Mellado B, Melichar B, Tomasek J, Kremer A, Kim HJ, Wood K, Dutcus C and Larkin J: Lenvatinib, everolimus, and the combination in patients with metastatic renal cell carcinoma: A randomised, phase 2, open-label, multicentre trial. Lancet Oncol 16(15): 1473-1482, 2015. PMID: 26482279. DOI: 10.1016/S14702045(15)00290-9

5 Motzer RJ, Tannir NM, McDermott DF, Aren Frontera O, Melichar B, Choueiri TK, Plimack ER, Barthelemy P, Porta C, George S, Powles T, Donskov F, Neiman V, Kollmannsberger CK, Salman P, Gurney H, Hawkins R, Ravaud A, Grimm MO, Bracarda S, Barrios CH, Tomita Y, Castellano D, Rini BI, Chen AC, Mekan S, McHenry MB, Wind-Rotolo M, Doan J, Sharma P, Hammers HJ, Escudier B and CheckMate I: Nivolumab plus ipilimumab versus sunitinib in advanced renal-cell carcinoma. $\mathrm{N}$ Engl J Med 378(14): 1277-1290, 2018. PMID: 29562145. DOI: 10.1056/NEJMoa1712126
6 Buder-Bakhaya K and Hassel JC: Biomarkers for clinical benefit of immune checkpoint inhibitor treatment-a review from the melanoma perspective and beyond. Front Immunol 9: 1474, 2018. PMID: 30002656. DOI: 10.3389/fimmu.2018.01474

7 Heng DY, Xie W, Regan MM, Warren MA, Golshayan AR, Sahi C, Eigl BJ, Ruether JD, Cheng T, North S, Venner P, Knox JJ, Chi KN, Kollmannsberger C, McDermott DF, Oh WK, Atkins MB, Bukowski RM, Rini BI and Choueiri TK: Prognostic factors for overall survival in patients with metastatic renal cell carcinoma treated with vascular endothelial growth factortargeted agents: Results from a large, multicenter study. J Clin Oncol 27(34): 5794-5799, 2009. PMID: 19826129. DOI: 10.1200/JCO.2008.21.4809

8 Eisenhauer EA, Therasse P, Bogaerts J, Schwartz LH, Sargent D, Ford R, Dancey J, Arbuck S, Gwyther S, Mooney M, Rubinstein L, Shankar L, Dodd L, Kaplan R, Lacombe D and Verweij J: New response evaluation criteria in solid tumours: Revised recist guideline (version 1.1). Eur J Cancer 45(2): 228247, 2009. PMID: 19097774. DOI: 10.1016/j.ejca.2008.10.026

9 Gershenwald JE, Scolyer RA, Hess KR, Sondak VK, Long GV, Ross MI, Lazar AJ, Faries MB, Kirkwood JM, McArthur GA, Haydu LE, Eggermont AMM, Flaherty KT, Balch CM, Thompson JF, for members of the American Joint Committee on Cancer Melanoma Expert P, the International Melanoma D and Discovery P: Melanoma staging: Evidence-based changes in the american joint committee on cancer eighth edition cancer staging manual. CA Cancer J Clin 67(6): 472-492, 2017. PMID: 29028110. DOI: $10.3322 /$ caac.21409

10 Motzer RJ, Bacik J, Murphy BA, Russo P and Mazumdar M: Interferon-alfa as a comparative treatment for clinical trials of new therapies against advanced renal cell carcinoma. J Clin Oncol 20(1): 289-296, 2002. PMID: 11773181. DOI: 10.1200/ JCO.2002.20.1.289

11 Powles T, Albiges L, Staehler M, Bensalah K, Dabestani S, Giles RH, Hofmann F, Hora M, Kuczyk MA, Lam TB, Marconi L, Merseburger AS, Fernandez-Pello S, Tahbaz R, Volpe A, Ljungberg B and Bex A: Updated european association of urology guidelines recommendations for the treatment of firstline metastatic clear cell renal cancer. Eur Urol, 2017. PMID: 29223605. DOI: 10.1016/j.eururo.2017.11.016

12 McDermott DF, Huseni MA, Atkins MB, Motzer RJ, Rini BI, Escudier B, Fong L, Joseph RW, Pal SK, Reeves JA, Sznol M, Hainsworth J, Rathmell WK, Stadler WM, Hutson T, Gore ME, Ravaud A, Bracarda S, Suarez C, Danielli R, Gruenwald V, Choueiri TK, Nickles D, Jhunjhunwala S, Piault-Louis E, Thobhani A, Qiu J, Chen DS, Hegde PS, Schiff C, Fine GD and Powles T: Clinical activity and molecular correlates of response to atezolizumab alone or in combination with bevacizumab versus sunitinib in renal cell carcinoma. Nat Med 24(6): 749757, 2018. PMID: 29867230. DOI: 10.1038/s41591-018-0053-3

13 Azuma M, Shi M, Danenberg KD, Gardner H, Barrett C, Jacques CJ, Sherod A, Iqbal S, El-Khoueiry A, Yang D, Zhang W, Danenberg PV and Lenz HJ: Serum lactate dehydrogenase levels and glycolysis significantly correlate with tumor vegfa and vegfr expression in metastatic crc patients. Pharmacogenomics $8(12)$ : 1705-1713, 2007. PMID: 18086000. DOI: $10.2217 / 14622416$. 8.12.1705

14 Giampieri R, Puzzoni M, Daniele B, Ferrari D, Lonardi S, Zaniboni A, Cavanna L, Rosati G, Pella N, Zampino MG, Sozzi P, Germano D, Zagonel V, Codeca C, Libertini M, Labianca R, 
Cascinu S, Italian Group for the Study of Gastrointestinal C and Scartozzi M: First-line folfiri and bevacizumab in patients with advanced colorectal cancer prospectively stratified according to serum ldh: Final results of the giscad (italian group for the study of digestive tract cancers) central (colorectalavastintrialldh) trial. Br J Cancer 117(8): 1099-1104, 2017. PMID: 28926529. DOI: $10.1038 /$ bjc 2017.234

15 van Dijk N, Funt SA, Blank CU, Powles T, Rosenberg JE and van der Heijden MS: The cancer immunogram as a framework for personalized immunotherapy in urothelial cancer. Eur Urol 75(3): 435-444, 2019. PMID: 30274701. DOI: 10.1016/j.eururo. 2018.09 .022

16 Shrotriya S, Walsh D, Bennani-Baiti N, Thomas S and Lorton $\mathrm{C}$ : C-reactive protein is an important biomarker for prognosis tumor recurrence and treatment response in adult solid tumors: A systematic review. PLoS One 10(12): e0143080, 2015. PMID: 26717416. DOI: 10.1371 /journal.pone. 0143080
17 Bilen MA, Dutcher GMA, Liu Y, Ravindranathan D, Kissick HT, Carthon BC, Kucuk O, Harris WB and Master VA: Association between pretreatment neutrophil-to-lymphocyte ratio and outcome of patients with metastatic renal-cell carcinoma treated with nivolumab. Clin Genitourin Cancer 16(3): e563-e575, 2018. PMID: 29402706. DOI: 10.1016/j.clgc.2017.12.015
Received May 14, 2019

Revised June 9, 2019

Accepted June 10, 2019 